\title{
How tall can gelatin towers be? An introduction to elasticity and buckling.
}

\author{
Nicolas Taberlet, * Jérémy Ferrand, Élise Camus, Léa Lachaud, and Nicolas Plihon \\ Univ Lyon, Ens de Lyon, Univ Claude Bernard, \\ CNRS, Laboratoire de Physique, F-69342 Lyon, France
}

(Dated: January 4, 2020)

\begin{abstract}
The stability of elastic towers is studied through simple hands-on experiments. Using gelatinbased stackable bricks, one can investigate the maximum height a simple structure can reach before collapsing. We show through experiments and using the classical linear elastic theory, that the main limitation to the height of such towers is the buckling of the elastic structures under their own weight. Moreover, the design and architecture of the towers can be optimized to greatly improve their resistance to self-buckling. To this aim, the maximum height of hollow and tapered towers is investigated. The experimental and theoretical developments presented in this paper can help students grasp at fundamental concepts in elasticity and mechanical stability.
\end{abstract}

\section{INTRODUCTION}

The stability of freestanding structures is of great importance in architecture and relies on simple physical principles. ${ }^{1-3}$ The main potential causes of collapse include misalignments of the structure and failure of the building material itself. However, when a tower is submitted to a compressive normal force, it can undergo sudden lateral deflections, a phenomenon known as buckling. ${ }^{4}$ In particular, the weight of the structure leads to non-uniform normal loads (more intense at the base of the tower than at its top), a phenomenon that will be refereed to as self-buckling in the remaining of the text. In this article, we propose to study this instability at the laboratory scale in simple, affordable, hands-on experiments using gelatin-based materials. The rigidity (or Young's modulus) of the material can be easily varied by changing the gelatin concentration and its consequences on the maximum height of a tower prior to buckling are spectacular. We show that the linear continuous theory of elasticity allows to precisely predict the maximum height of long gelatin bars (of square or circular cross section). We introduce a useful trick to repeat over and over the experiments using individual gelatin-based stackable bricks, produced using a home-made silicone mold of classical Lego ${ }^{\circledR}$ bricks, which allows them to firmly interlock as displayed in Fig. 6. The usefulness of this method relies on the failure mode of the tower when it exceeds the maximum stable height (see the rightmost photograph of Fig. 6): while irreversible fractures appear within a solid block, individual bricks simply detach in the brick tower (and may be used again for further studies). Moreover, the versatility of the gelatin bricks allows one to investigate the increase in stability of smartly-designed freestanding structures.

The experimental protocol described in this article provides an interesting hands-on illustration of the theory of elasticity, more specifically to the Euler-Bernoulli theory for beam deflection, ${ }^{5-9}$ and to the phenomenon of buckling. The results presented in this article may also be used as an illustration of the importance of

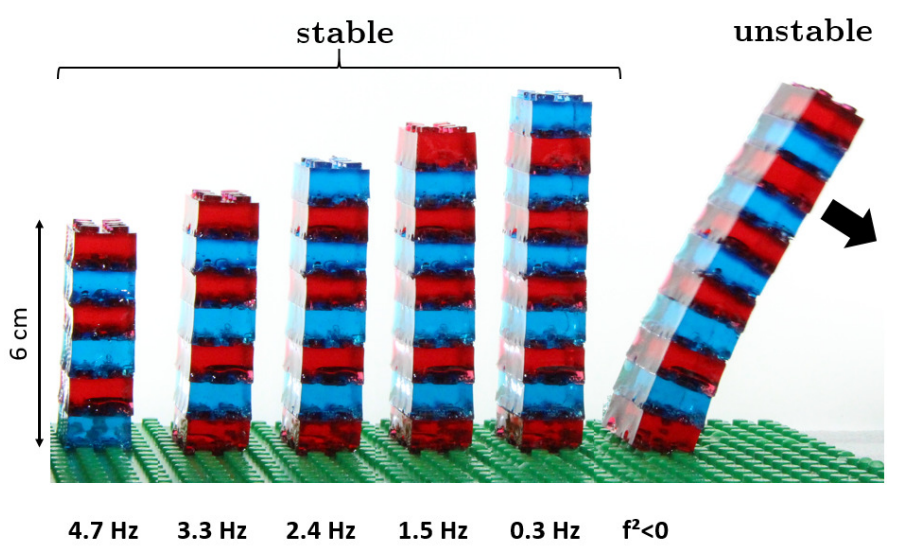

FIG. 1. Stability of gelatin-based Lego ${ }^{\circledR}$-brick towers. Below a critical height $(10.5 \pm 0.5 \mathrm{~cm}$ for a gelatin concentration of $16 \%$ ), when perturbed, a tower oscillates around its stable vertical equilibrium position (at the indicated frequency). Above the critical height, any small-amplitude perturbation causes the tower to collapse (right-most tower).

dimensional analysis. ${ }^{10-12}$ Moreover, we show that the predicted frequency of the free oscillations (obtained through a hand-waving argument rather than a rigorous and unnecessarily complex derivation) shows excellent agreement with the experimental measurements.

The article is organized as follows. In section II, the framework of the linear theory of elasticity is recalled, a dimensional analysis of self-buckling is proposed, theoretical predictions for the critical self-buckling height are derived, and the frequency of oscillations of stable towers are theoretically investigated. Section III presents experimental results on the mechanical properties of the gelatin-based gels, on the maximum height of stable towers as well as on the frequency of free oscillations. Finally, the increase in stability of hollow or tapered towers is discussed in section IV. 


\section{THEORY OF SELF BUCKLING FROM ELASTICA}

This section recalls and derives theoretical predictions of the critical self-buckling height and of the oscillation frequency of elastic beams using a continuum approach. The relevance of this approach is shown in Sec. IIIB: the critical self-buckling height measured of long gelatinbased blocks is identical to that of towers made from stacked bricks (and having the same geometry).

\section{A. Euler-Bernoulli beam theory}

The bending and buckling of an elastic tower is well described by the Euler-Bernoulli beam theory, which is a simplification of the linear elasticity theory applied to the case of small deflections. ${ }^{13}$ This theory postulates the existence of a neutral fiber (at the center of the beam), whose length remains unchanged and which remains locally perpendicular to the cross section of a bent beam. The essential result of this theory is that there exists a linear relation between the bending moment, $M$, within the beam and its local curvature, $\kappa$ :

$$
M=E I \kappa
$$

where $E$ is the Young's modulus and $I=\iint r^{2} d A$ is the second moment of area, known as the area moment of inertia in engineering ( $r$ being the distance to the neutral fiber and $d A$ the surface element in the cross section). In the case of a rectangular beam of thickness $w$ and depth $d$ (where by convention $w<d$ ), $I=w^{3} d / 12$ and the surface area of the cross section is $A=w d .{ }^{14}$

\section{B. Dimensional analysis}
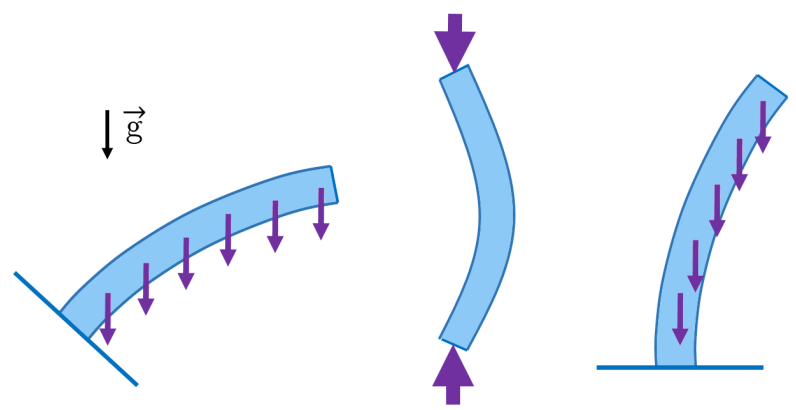
a) bent
b) buckled
c) self-buckled

FIG. 2. Schematics of elastic beams. a) oblique beam bent under its own weight, b) buckled tip-loaded beam and c) clamped beam self-buckled under its own weight. The first case causes a continuous deformation of the beam while the two others display a well-defined threshold and lead to sudden large-amplitude deformations.
Let us first consider the case of a narrow strip of paper (or a thin metal plate, or a plastic sheet) representing the beam and held between two fingers at an angle of $45^{\circ}$ (Fig. 7 a). If the strip is short enough (typically less than $2 \mathrm{~cm}$ ) it appears to remain perfectly straight while a longer strip (typically $20 \mathrm{~cm}$ ) is completely bent under its own weight. There is therefore a clear competition between the flexural rigidity $(E I)$ and the weight (per unit length) $\rho g A$, where $\rho$ is the density of the material and $g$ the standard gravity), the ratio of which has the dimension of a length cubed. One can therefore define, from dimensional analysis, a characteristic length

$$
L_{c}=\left(\frac{E I}{\rho g A}\right)^{1 / 3}
$$

For typical paper $\left(w=0.1 \mathrm{~mm}, \rho=800 \mathrm{~kg} / \mathrm{m}^{3}, E=3 \mathrm{GPa}\right)$, $L_{c} \simeq 7 \mathrm{~cm}$, which corresponds to the simple experiments described above. Therefore, $L_{c}$ can be seen as a critical length above which an oblique beam noticeably bends under its own weight.

\section{Buckling and self-buckling}

The case of a vertical beam is more subtle. If the elastic tower is initially perfectly vertical (however tall), its own weight exerts no moment and cannot cause the beam to bend or buckle. However, a straight beam may become unstable to lateral deflections above a critical load, known as Euler's critical load (in the case of a tower, corresponding to a critical height). ${ }^{4}$ Buckling is thus an instability with a well-defined threshold (or bifurcation).

When the total load is located at the tip of a vertical tower (therefore neglecting the weight of the tower itself), the critical load above which a tower will buckle when submitted to any perturbation depends on the boundary conditions. For a beam of height $H$ clamped at its base and free at its top, the critical load $F_{c}$ is given by: ${ }^{4}$

$$
F_{c}=\left(\frac{\pi}{2}\right)^{2} \frac{E I}{H^{2}}
$$

The case of self-buckling, i.e. where the load is uniformly distributed along its height (and with no load at the top) is mathematically somewhat more complex but physically very similar to Euler's critical load, and was solved as early as the XVIII ${ }^{t h}$ century. ${ }^{15}$ The critical height is given by: ${ }^{16}$

$$
H_{c}=\left(\left(\frac{3 j_{1 / 3}}{2}\right)^{2} \frac{E I}{\rho g A}\right)^{1 / 3} \simeq 1.986\left(\frac{E I}{\rho g A}\right)^{1 / 3}
$$

where $j_{1 / 3} \simeq 1.866$ is the first zero of the $J_{1 / 3}$ Bessel function. Below this height, when shaken a tower simply oscillates around the stable vertical position, whereas above this height, any small perturbation will cause the 
structure to fail. Note that the height $H_{c}$ only differs from the one obtained by dimensional analysis (see Eq. 2) by a numerical factor.

As a side-note, from Eq. 3 and Eq. 4 one can see that the critical height of a tower whose load is uniformly distributed is $3 j_{1 / 3} / \pi \simeq 1.78$ times that of the same beam if its whole weight $\rho g A H_{c}$ were placed at the top.

\section{Oscillations of a vertical beam clamped at its base}

An elastic beam hanging from the ceiling is somewhat reminiscent of a pendulum pushed back to the vertical position by a linear spring: it can oscillate around its vertical equilibrium position due to elasticity (with an angular frequency $\omega_{e}$, if the weight is neglected) and due to gravity (with an angular frequency $\omega_{g}$ if the elasticity is neglected). These two independent frequencies are given by: ${ }^{14,17}$

$$
\left\{\begin{array}{l}
\omega_{e}^{2}=\left(\frac{\beta_{1}}{H}\right)^{4} \frac{E I}{\rho A} \\
\omega_{g}^{2}=\left(\frac{j_{0}}{2}\right)^{2} \frac{g}{H}
\end{array}\right.
$$

where $\beta_{1} \simeq 1.875$ is the first zero of $\cos \cosh +1$ and $j_{0} \simeq$ 2.405 , the first zero of the $J_{0}$ Bessel function. In the case of the spring-pendulum, for small-amplitude oscillations the system is linear and the resulting angular frequency is simply given by: $\omega^{2}=\omega_{e}^{2}+\omega_{g}^{2}$. As a crude first-order assumption, one can consider that the previous linear calculation still holds for the hanging elastic beam. In the case of a vibrating tower whose height is less than the critical self-buckling height, the weight has a destabilizing effect and the angular frequency of the small-amplitude oscillations is now given by:

$$
\omega^{2}=\omega_{e}^{2}-\omega_{g}^{2}
$$

The frequency vanishes to zero when the height of a tower reaches:

$$
H_{c}=\left(\left(\frac{2 \beta_{1}^{2}}{j_{0}}\right)^{2} \frac{E I}{\rho g A}\right)^{1 / 3} \simeq 2.045\left(\frac{E I}{\rho g A}\right)^{1 / 3}
$$

Thus, this equation is not strictly identical to Eq. 4, but only differ by the expression of the prefactor. However the numerical values of the two prefactors are very close (by less than 3\%), which indicates that the crude model proposed for the oscillation frequency is relevant. Experimental measurements of the frequency are discussed in section III C and are in good accordance with Eq. 7.

\section{EXPERIMENTS USING GELATIN-BASED TOWERS}

\section{A. Mechanical properties of the gelatin gel}

The experimental investigation reported below were carried out using blocks or bricks made of gelatin, an affordable and safe visco-elastic material. Gelatin is a gelling agent made of hydrolyzed collagen and obtained from skin, bones and connective tissues of pigs, chickens, cows and fish. ${ }^{18}$ It forms an elastic gel when dissolved in hot water and left to cool. The mechanical properties of the gel depends on their mass concentration in gelatin $C$, on the preparation protocol (most importantly on the duration and temperature at which the gel sets), as well as the initial gelation strength of the dry gelatin (characterized by the standardized Bloom number test). ${ }^{19}$ The gelatin used in our experiments has a Bloom number ranging from 200 to 225 . The mechanical properties of gelatin gels are very sensitive to temperature changes: typically, a variation in $2 C^{\circ}$ of the ambient temperature may cause a $20 \%$ change in the Young's modulus. ${ }^{20}$ When exposed to air gelatin samples quickly dry out and must therefore be kept wet during experiments. Moreover they may slowly swell when stored in water over several days. All gelatin blocks or bricks were produced using a home-made molds and where left to set in a fridge for $24 \mathrm{~h}$ prior to the experiments, conducted at $20 C^{\circ}$. The density of the gels (for concentrations up to $30 \%$ ) does not significantly differ from that of pure water. Note that agar-agar gels set within minutes and can represent a good alternative to gelatin-based gels.

The Young's modulus, $E$, of the gelatin gels and their compressive strength, $\sigma_{c}$, (the pressure at which they fail, forming irreversible fractures) were measured using an Anton Paar AR1000 rheometer in which cylindrical samples (20 mm in height and diameter) were tested. This device can simultaneously measure the applied normal force (with an accuracy of $10^{-4} \mathrm{~N}$ ) and the resulting deformation (with an accuracy of less than $1 \mu \mathrm{m}$ ). The Young's modulus is measured as the initial slope of the stress-strain curve while the compressive strength is computed from the maximum stress sustained before the sample fractures.

Figure 8 shows the results as a function of the concentration at a temperature of $20 C^{\circ}$. The Young's modulus ranges from 0 for $C \simeq 3.5 \%$, (meaning that the solution is simply liquid at low concentrations) to $75 \mathrm{kPa}$ for $C=20 \%$. Note that the values of $E$ and $\sigma_{c}$ are of the same order of magnitude, which indicates that the material can undergo very large deformations (more than $50 \%$ ) before failing.

Let us emphasize that the high precision of a commercial rheometer is an unnecessary luxury since, as mentioned above, the mechanical properties of the gel are very sensitive to the concentration, the preparation protocol and to small temperature variations. The measurements of $E$ and $\sigma_{c}$ can be easily performed by compress- 


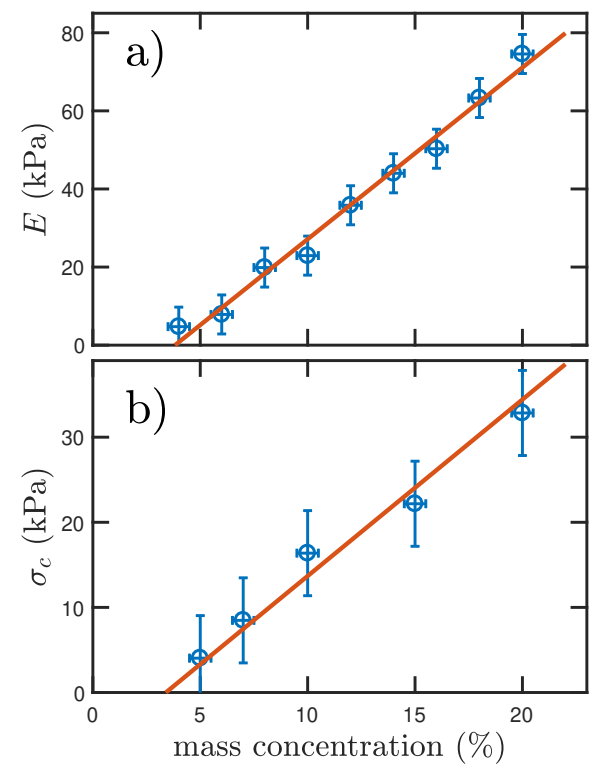

FIG. 3. Young's modulus, $E$, and compressive strength, $\sigma_{c}$ of gelatin gels at $20 C^{\circ}$ as a function of the weight concentration in gelatin. Both quantities displays a linear dependence on the concentration, and vanish at $C=3.5 \pm 0.5 \%$, indicating that below this critical concentration, the medium can be considered liquid. The solid lines are linear fits.

ing a sample directly on a scale while the deformation is measured with a caliper.

\section{B. Maximum height of simple towers before self-buckling}

Before introducing the use of stackable bricks made of gelatin, we first report the maximum height of a continuous beam made of gelatin. Two blocks $(16 \times$ $16 \mathrm{~mm}$ and $32 \times 32 \mathrm{~mm}$ ) of concentration $14 \%$ were used and their height prior to buckling is reported in Fig. 9 (open circles). Clearly, these two points align well with the other data sets obtained with stacked bricks. This confirms that the behavior of towers made of individual bricks is identical (when it comes to the critical self-buckling height) to that of solid tower of gelatin gels, and it validates the continuum approach used in Sec. II. Note however that for large deformations (far above the critical height) the solid blocks may experience irremediable fracturing whereas individual bricks can simply separate and be reused.

The bricks were produced using a home-made silicone mold of classical Lego ${ }^{\circledR}$ bricks. We used bricks of dimensions width $w=16 \mathrm{~mm}$, depth $d=32 \mathrm{~mm}$ and height 10 $\mathrm{mm}$, showing 2 rows of 4 studs, further referred to as $4 \times 2$ bricks. Cutting those in half creates $4 \times 1$ bricks while interlocking them, one can build wider towers. Individual bricks are carefully stacked up until the tower falls. Us- ing this simple protocol, the maximum height $H_{\max }$ is bounded between two integer values (between 10 and 11 bricks on Fig. 6 , i.e. $10 \mathrm{~cm}<H_{\max }<11 \mathrm{~cm}$ ). Figure 9 shows the results obtained for $4 \times 1,4 \times 2$ and $4 \times 4$ towers, and for three concentrations $C=8 \%, C=12 \%$ and $C=16 \%$. The corresponding maximum heights range from $4 \mathrm{~cm}$ to $16 \mathrm{~cm}$. The experimental measurements are plotted as a function of the theoretical predictions of Eq. 4, using the values of the Young's modulus of Fig. 8.

This results validates the continuous medium approach used for the tower made from stacked bricks, indicating again that a tower made of individual bricks will buckle at the same height as a solid block of gel of identical cross section.

Note however that if the cross section is large enough, the compressive strength may become the limiting factor. Indeed, the maximum height before the bottom brick of the tower fails is given by the hydrostatic pressure: $\rho g H=\sigma_{c}$. Nevertheless, for a concentration of $C=8 \%$ the corresponding height is as large as $90 \mathrm{~cm}$ whereas it reaches $350 \mathrm{~cm}$ for $C=20 \%$. Let us also mention that for such tall towers, the irregularities in the shape of the individual bricks can cause the tower to simply tip over since its center of gravity might not remain above its base.

\section{Predicting self buckling from stable structures self-oscillations}

The oscillatory motion of stable $4 \times 2$ towers, at a concentration of $C=16 \%$ (as in Fig. 6), was studied using a camera (Ximea, xiQ MQ013MG-ON). The tower is given an initial gentle push (of less than a $\mathrm{cm}$ ) and its free oscillations are recorded at $100 \mathrm{~Hz}$. The period is computed from the average duration of the first 5 oscillations yielding an estimated uncertainty of $0.1 \mathrm{~Hz}$. Figure 10

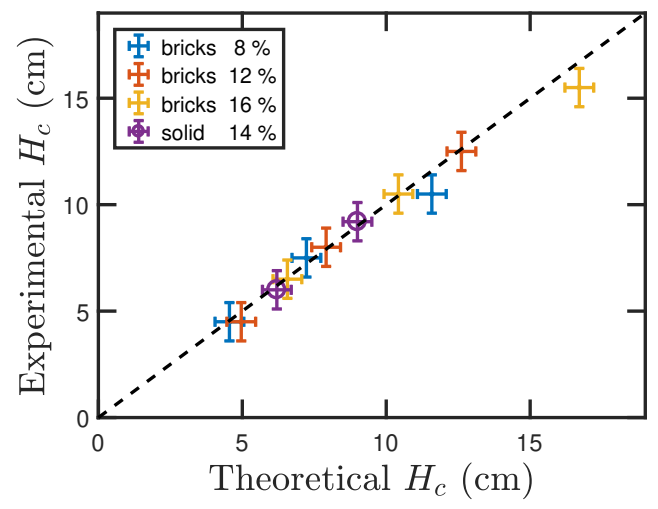

FIG. 4. Experimental vs. theoretical critical self-buckling height, for towers of rectangular cross-sections, for various dimensions and gelatin concentrations. The solid black line has a slope of 1 and emphasizes the excellent agreement between the experimental data and the theoretical predictions. 
presents the results obtained for towers of height $H$ ranging from $5 \mathrm{~cm}$ to $10 \mathrm{~cm}$. The frequency is plotted as a function of $H$ on panel a) and of $1 / H^{2}$ in panel b) (since the frequency of a beam whose weight is neglected scales as $1 / H^{2}$, as indicated by Eq. 5).
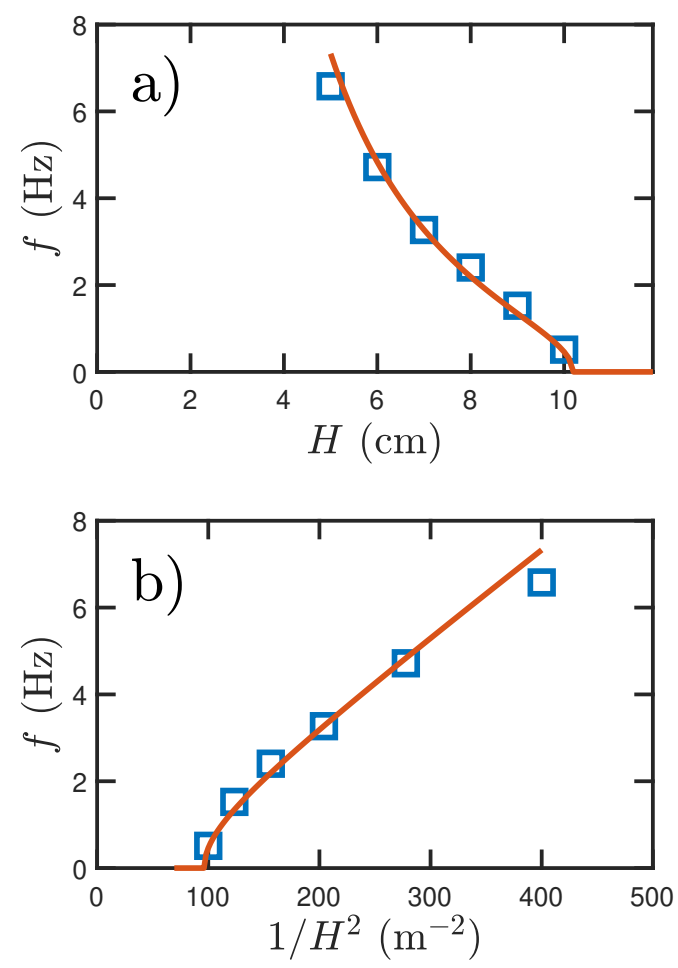

FIG. 5. Frequency, $f$, of the free oscillations of a tower $(4 \times 2$ , $C=16 \%$ ) (a) as a function of its height, $H$, (b) as a function of $1 / H^{2}$. The solid line is the theoretical prediction of Eq. 6. The frequency vanishes to zero as the height tends toward the critical self-buckling height. The good agreement between the experimental data and the theory validates the hypotheses made and shows that self-buckling is indeed the main limitation to the height of gelatin towers.

On both panels, the red line corresponds to the simplified model presented in section II D and perfectly matches the experimental data. Again, this indicates that the limiting factor in the height of a tower originates from the competition between its elasticity (a stabilizing effect) and its own weight (a destabilizing effect).

Let us mention that the measurement of the frequency of the free oscillations can also constitute a good tool to accurately determine the critical self-buckling height. ${ }^{21}$ Stacking up individual bricks until the tower buckles only lead to a precision of one brick height (1 $\mathrm{cm}$ in our case) whereas the frequency vs. $H$ might simply be interpolated as a straight line (when vanishing to zero) whose intersect with the $x$-axis gives a value of $H_{c}$ with a typical accuracy of $1 \mathrm{~mm}$.

\section{TOWARD HIGH TOWERS: INFLUENCE OF DESIGN AND SHAPES}

The shape of most actual towers and sky-scrappers is not simply rectangular: from the pyramids of Egypt in ancient times, to the more modern Eiffel tower and Burj Khalifa, architects have always conceived creative designs. The choice of complex shapes is not entirely aesthetic (or financial) but is often governed by physical and engineering considerations. ${ }^{1,22}$ Two major improvements can be done to increase the critical self-buckling height of an elastic tower: the structure can be hollow or tapered. In this section, these two effects are studied through experiments and theoretical predictions. Note however that although a hollow structure could reach a greater self-buckling height, its overall ability to support an additional external weight could be less than the corresponding solid tower.

Let us mention that the biggest challenges modern skyscrappers have to face include buckling and oscillating under strong winds and resistance to earth-quakes, rather than buckling under their own weight. ${ }^{23}$

\section{A. Hollow towers}

It is obvious from Eq. 4 that increasing the second moment of area $I$ (while the mass per unit length is kept constant) pushes back the self-buckling limitation. A simple experiment can be made to demonstrate the increase in stability of hollow towers. Using $4 \times 1$ bricks, one can build a simple $4 \times 4$ tower and a hollow $5 \times 5$ tower whose cross-sections areas are equal (see Fig. 11). The ratio of the second moment of area of the two structures is therefore $\left(5^{4}-3^{4}\right) / 4^{4}=2.125$, showing an outstanding increase in the rigidity of the tower. The critical height of the hollow tower is thus $2.13^{1 / 3} \simeq 1.29$ times that of the full structure. The difference between the two is clearly visible in Fig. 11, in which the ratio of the two heights corresponds to the predicted value.

The stability of two types of hollow towers built using a variety of concentrations was investigated: the $5 \times 5$ hollow tower displayed in Fig. 11 of width $4 \mathrm{~cm}$ (which should be compared to a solid $4 \times 4$ tower, which has the same material per unit length), and a $9 \times 9$ hollow tower (made of $4 \times 1$ bricks, with a $7 \times 7$ empty hole in the center) of width $7.2 \mathrm{~cm}$ (which reached $38 \mathrm{~cm}$ in height!). The results are summarized in table I and are in very good accordance with the theoretical predictions.

Another interesting consequence of Eq. 4 is the comparison with a solid tower of identical width. If a fraction $\phi$ of a tower is hollow so that its surface area scales as $1-\phi^{2}$ and its second moment of area as $1-\phi^{4}$, then the corresponding maximum height scales as $\left(1+\phi^{2}\right)^{1 / 3}$, which ranges from 1 to $2^{1 / 3} \simeq 1.260$. It therefore appears that thinner walls allow for taller structures. However, another limitation arises as the walls thin down: for wide structures and thin walls, the walls themselves may start 
TABLE I. Critical self-buckling height of solid and hollow towers built using $4 \times 1$ bricks for various concentrations and overall widths.

\begin{tabular}{ccccc}
\hline \hline $\begin{array}{c}\mathrm{C} \\
\%\end{array}$ & $\begin{array}{c}\text { Total width } \\
\mathrm{cm}\end{array}$ & $\begin{array}{c}H_{c} \text { (solid) } \\
\mathrm{cm}( \pm 0.5)\end{array}$ & $\begin{array}{c}H_{c} \text { (hollow) } \\
\mathrm{cm}( \pm 0.5)\end{array}$ & $\begin{array}{c}\text { Theory (hollow) } \\
\mathrm{cm}( \pm 0.5)\end{array}$ \\
\hline 10 & 4.0 & 12.5 & 16.5 & 16.1 \\
30 & 4.0 & 20.5 & 25.5 & 25.3 \\
30 & 7.2 & 25.5 & 38 & 36.5 \\
\hline \hline
\end{tabular}

to buckle under the weight of the structure. The determination of the optimal thickness of the wall is a complex problem with no analytical solution. ${ }^{24-26}$

\section{B. Tapered towers}

The principle behind the increased rigidity of tapered tower seem simple since most of its weight is located at the bottom. Yet, the rigidity of such a tower also decreases as the structure tappers, and an exact calculation of their critical self-buckling height, $H_{c}$, is necessary. ${ }^{27}$

We need to mention that using individual bricks, it can be difficult to build smooth tapered towers. Therefore, in this section experiments were conducted using solid wedges and pyramids of gelatin gels, directly molded in home-made molds. The critical height for self-buckling is then measured when holding carefully the base of the tapered structure using both hands, which results is rather large uncertainties (typically 2 $\mathrm{cm})$, but nevertheless proves accurate.

\section{Wedges}

For a wedge of half-angle $\alpha$ (long in the perpendicular direction), the critical height is given by:

$$
H_{c}^{\text {wedge }}=\frac{j_{2}^{2}}{6} \frac{E}{\rho g} \tan ^{2} \alpha
$$

where $j_{2} \simeq 5.136$ is the first zero of the $J_{2}$ Bessel function. The critical height can be rewritten as a function of the corresponding width at the base given by $a=2 H_{c}^{\text {wedge }} \tan \alpha$ :

$$
H_{c}^{\text {wedge }}=\left(\frac{j_{2}^{2}}{2} \frac{E a^{2}}{12 \rho g}\right)^{1 / 3}
$$

In comparison, for a simple rectangular tower of width $a$ (see Eq. 4):

$$
H_{c}^{\text {rect. }}=\left(\left(\frac{3 j_{1 / 3}}{2}\right)^{2} \frac{E a^{2}}{12 \rho g}\right)^{1 / 3}
$$

Equations 9 and 10 indicate that a wedge-shaped tapered tower can be 1.190 times as tall as a rectangular tower of identical width at its base.
Figure 12a shows the experimental maximum height before self-buckling for wedges of various half-angles and for various concentrations. The experimental data is plotted as a function of the corresponding theoretical value of $H_{c}^{\text {rect. }}$ for rectangular towers and the dashed-line has the expected slope of 1.190. The agreement between the experiments and the prediction is very satisfactory, showing that all wedge-shaped towers are indeed $\simeq 19 \%$ taller than their rectangular counterparts.

\section{Pyramid}

The shape can be further improved by building a tower which tapers down towards the tip in both directions, its cross-section being a square as opposed to a rectangle in the case of a wedge. The critical height of such a pyramid of half-angle $\alpha$ is given by: ${ }^{16}$

$$
H_{c}^{\text {pyramid }}=\left(\frac{j_{3}}{2}\right)^{2} \frac{E}{\rho g} \tan ^{2} \alpha
$$

where $j_{3} \simeq 6.380$ is the first zero of the $J_{3}$ Bessel function.

For the same half-angle, the height of a pyramid can therefore be as much as $\left(3 j_{3}^{2}\right) /\left(2 j_{2}^{2}\right) \simeq 2.31$ times that of a wedge-shaped tower. The maximum height of pyramids and wedges of identical half-angles were experimentally measured for various values of $\alpha$ and various concentrations. Figure $12 \mathrm{~b}$ shows a comparison between the two shapes as the slope of the dashed line is the predicted ratio. Note that pyramids can be $2.31^{1 / 3} \simeq 1.32$ as tall as the corresponding wedge-shaped towers of identical widths.

\section{CONCLUSION}

We have shown that the main limiting factor of the stability of gelatin-based brick towers is the buckling under their own weight. The classical theory of elasticity provides a continuum approach whose predictions (of the critical height and of the frequency of the oscillations of stable tower) are in good agreement with experimental measurement. Two improvements that can help push the limits of self-buckling are proposed: structures can be hollow or tapered. Combining both techniques, we were able to build a brick tower (pyramidal and hollow, $12 \times 12$ at its base, $C=30 \%$ ) as tall as $56 \mathrm{~cm}$.

As a digression, the mechanical properties of the gels could be further investigated. Indeed, gelatin-based gels are not simply elastic but display a viscoelastic behavior (reminiscent of the properties of silly putty: ${ }^{28}$ ) the storage modulus (which characterizes the elasticity) is typically larger than the loss modulus (which characterizes the viscous behavior) but for low concentrations, both quantities are of the same order of magnitude. ${ }^{29} \mathrm{Al}-$ though, as a first-order approximation, the material can be considered purely elastic, the bricks can undergo a slow deformations when submitted to a constant pressure 
(a phenomenon known as creep flow ${ }^{30,31}$ typically occurring within a few minutes). These properties can affect the stability of the brick-towers. Stacks whose height is less than but close to the expected critical height for self-buckling might be initially stable when built, but can slowly become unstable and buckle within minutes.

\section{ACKNOWLEDGMENTS}

The authors acknowledge support from the University of Lyon, the University Lyon Claude Bernard, the École
Normale Supérieure de Lyon and its Physics Department and Laboratoire de Physique. We are thankful to J. Bernard, E. Camus, A. Gayout, T. Guyomar, F. Museur and $\mathrm{L}$. Thorens for fruitful discussions.
* nicolas.taberlet@ens-lyon.fr

1 W. Addis, Building: 3000 years of design engineering and construction. Phaidon Press, 2007.

2 B. N. Sandaker, A. P. Eggen, and M. R. Cruvellier, The structural basis of architecture. Routledge, 2013.

3 F. Moore, Understanding structures. McGraw-Hill Science Engineering, 1999.

4 R. M. Jones, Buckling of bars, plates, and shells. Bull Ridge Corporation, 2006.

${ }^{5}$ L. D. Landau, L. P. Pitaevskii, A. M. Kosevich, and E. M. Lifshitz, Theory of Elasticity, Third Edition: Volume 7 (Course of Theoretical Physics). Butterworth-Heinemann, 3 ed., 1986.

6 R. D. Edge, "Elasticity," Phys. Teach. 21 (9), 608-608 (1983).

7 J. Casey, "The elasticity of wood," Phys. Teach. 31 (5), 286-288 (1993).

8 K. Turvey, "An undergraduate experiment on the vibration of a cantilever and its application to the determination of young's modulus," Am. J. Phys. 58 (5), 483-487 ( 1990).

9 T. Hopfl, D. Sander, and J. Kirschner, "Demonstration of different bending profiles of a cantilever caused by a torque or a force," Am. J. Phys. 69 (10), 1113-1115 (2001).

${ }^{10}$ H. E. Huntley and J. V. Kline, "Dimensional analysis," Am. J. Phys., 24 (7), 534-535 (1956).

11 J. Palacios and H. L. Armstrong, "Dimensional analysis," Am. J. Phys., 33 (6), 513-514 (1965).

12 W. J. Remillard, "Applying dimensional analysis," Am. J. Phys., 51 (2), 137-140 (1983).

13 O. Bauchau and J. Craig, "Euler-bernoulli beam theory," in Struc. Ana. ), 173-221, Springer, 2009.

14 L. D. Landau, E. M. Lifshitz, L. Pitaevskii, and A. Kosevich, Course of Theoretical Physics: Volume 7, Theory of Elasticity. Pergamon Press, 1986.

15 W. Gautschi, "Leonhard euler: his life, the man, and his works," SIAM Rev. 50 (1), 3-33 (2008).

16 A. G. Greenhill, "Determination of the greatest height consistent with stability that a vertical pole or mast can be made, and of the greatest height to which a tree of given proportions can grow, https://martingillie.files.wordpress.com/2013/11/longestcolumn.pdf," Proc. Cam. Phil. Soc. 4, 65-73 (1881).

17 G. N. Watson, A treatise on the theory of Bessel functions. Cambridge university press, 1995.

18 I. J. Haug, K. I. Draget, and O. Smidsrød, "Physical and rheological properties of fish gelatin compared to mam-

FIG. 6. Stability of gelatin-based Lego ${ }^{\circledR}$-brick towers. Below a critical height $(10.5 \pm 0.5 \mathrm{~cm}$ for a gelatin concentration of $16 \%$ ), when perturbed, a tower oscillates around its stable vertical equilibrium position (at the indicated frequency). Above the critical height, any small-amplitude perturbation causes the tower to collapse (right-most tower).

malian gelatin," Food Hydr. 18 (2), 203-213 (2004).

19 F. A. Osorio, E. Bilbao, R. Bustos, and F. Alvarez, "Effects of concentration, bloom degree, and ph on gelatin melting and gelling temperatures using small amplitude oscillatory rheology," Int. J. Food Prop. 10 (4), 841-851 (2007).

20 M. Djabourov, J. Leblond, and P. Papon, "Gelation of aqueous gelatin solutions. ii. rheology of the sol-gel transition," Journal de Physique 49 (2), 333-343 (1988).

21 R. H. Plaut and L. N. Virgin, "Use of frequency data to predict buckling," J. Eng. Mech. 116 (10), 2330-2335 (1990).

22 G. J. Salamo, J. A. Brewer, and J. A. Berry, "Physics for architects," Am. J. Phys. 47 (1), 24-28 (1979).

23 J. Gallant, "The shape of the eiffel tower," Am. J. Phys. 70 (2), 160-162 (2002).

24 J. Rhodes, "Buckling of thin plates and members - and early work on rectangular tubes," Thin-Walled Struc. 40 (2), 87-108 (2002).

25 B. Schafer, "Local, distortional, and euler buckling of thinwalled columns," J. Struc. Eng. 128 (3), 289-299 (2002).

26 J. B. Keller, "The shape of the strongest column," Arch. Rat. Mech. Ana. 5 (1), 275-285 (1960).

27 W. G. Smith, "Analytic solutions for tapered column buckling," Comp. Struc. 28 (5), 677-681 (1988).

28 R. Cross, "Elastic and viscous properties of silly putty," Am. J. Phys. 80 (10), 870-875 (2012).

29 C. Michon, G. Cuvelier, and B. Launay, "Concentration dependence of the critical viscoelastic properties of gelatin at the gel point," Rheo. Acta 32 (1), 94-103 (1993).

30 P. Oswald, "Rheophysics," Rheophysics, by Patrick Oswald, Cambridge, UK: Cambridge University Press, 2009.

31 E. D. Zanotto, "Do cathedral glasses flow?," Am. J. Phys. 66 (5), 392-395 (1998). 
FIG. 7. Schematics of elastic beams. a) oblique beam bent under its own weight, b) buckled tip-loaded beam and c) clamped beam self-buckled under its own weight. The first case causes a continuous deformation of the beam while the two others display a well-defined threshold and lead to sudden large-amplitude deformations.

FIG. 8. Young's modulus, $E$, and compressive strength, $\sigma_{c}$ of gelatin gels at $20 C^{\circ}$ as a function of the weight concentration in gelatin. Both quantities displays a linear dependence on the concentration, and vanish at $C=3.5 \pm 0.5 \%$, indicating that below this critical concentration, the medium can be considered liquid. The solid lines are linear fits.

FIG. 9. Experimental vs. theoretical critical self-buckling height, for towers of rectangular cross-sections, for various dimensions and gelatin concentrations. The solid black line has a slope of 1 and emphasizes the excellent agreement between the experimental data and the theoretical predictions.

FIG. 10. Frequency, $f$, of the free oscillations of a tower $(4 \times 2, C=16 \%)$ (a) as a function of its height, $H$, (b) as a function of $1 / H^{2}$. The solid line is the theoretical prediction of Eq. 6. The frequency vanishes to zero as the height tends toward the critical self-buckling height. The good agreement between the experimental data and the theory validates the hypotheses made and shows that self-buckling is indeed the main limitation to the height of gelatin towers.

FIG. 11. Picture of solid and hollow towers (built from $4 \times 1$ bricks at $C=10 \%$ at their maximum height. The solid tower reaches $12.5 \pm 0.5 \mathrm{~cm}$, while the hollow tower reaches $16.5 \pm 0.5$ $\mathrm{cm}$, which corresponds to the predicted increase of $29 \%$ for hollow towers of identical cross-section areas.

FIG. 12. a) Experimental maximum height of wedge-shaped tower vs. the corresponding predicted critical height of rectangular tower of identical width at their base. The solid line shows the predicted proportionality (slope of 1.190) and nicely matches the data. As expected, wedges can be $19 \%$ taller than rectangular towers before buckling. b) Experimental maximum height of pyramidal towers vs. that of wedge-shaped tower of identical half-angle. The solid line shows the predicted proportionality (slope of 2.31), in excellent agreement with the experimental data. Pyramids can be $131 \%$ taller than wedges. 